Pacific Journal of Mathematics

MASS OF RAYS ON COMPLETE OPEN SURFACES 


\title{
MASS OF RAYS ON COMPLETE OPEN SURFACES
}

\author{
K. Shiohama, T. Shioya, and M. Tanaka
}

The total curvature of a complete open surface describes certain properties of the Riemannian structure which defines it. We study relationships between the total curvature and the mass of rays on a finitely connected complete open surface and obtain some integral formulas.

0. Introduction. Throughout this paper let $M$ be a connected, finitely connected, oriented, complete and noncompact Riemannian 2-manifold without boundary. The total curvature $c(M)$ of $M$ is defined to be an improper integral over $M$ of Gaussian curvature $G$ with respect to the area element $d M$ of $M$. A well-known theorem due to Cohn-Vossen [1] states that if $M$ admits total curvature, then $2 \pi \chi(M)-c(M) \geq 0$, where $\chi(M)$ is the Euler characteristic of $M$. Clearly $c(M)$ depends on the choice of Riemannian metric. This phenomenon gives rise to the idea that the value $2 \pi \chi(M)-c(M)$ should describe certain properties of Riemannian metric which defines it.

A ray (respectively, a straight line) on $M$ is by definition a unit speed geodesic parametrized on $[0, \infty)$ (respectively, on $\mathbb{R}$ ) every subarc of which realizes distance between its terminal points. For a point $p \in M$ let $S_{p}(1)$ be the unit circle centered at the origin of the tangent space $M_{p}$ to $M$ at $p$. Let $A(p)$ be the set of all unit vectors tangent to rays emanating from $p . A(p)$ is closed in $S_{p}(1)$. Let $\mathfrak{M}$ be the natural measure on $S_{p}(1)$ induced from the Riemannian metric. A relation between the mass of rays and the total curvature was first investigated by Maeda in [6], [7]. He proved that if $M$ is homeomorphic to $R^{2}$ and if $G \geq 0$, then $\mathfrak{M} \circ A \geq 2 \pi-c(M)$, and in particular $\inf _{M} \mathfrak{M} \circ A=2 \pi-c(M)$. These results were extended by Shiga in [10], [11] to Riemannian planes whose Gaussian curvatures change sign, and later by Oguchi [9] to finitely connected $M$ with one endpoint. In connection with an isoperimetric problem discussed by Fiala [3] and Hartman [4], the first-named author proved in [14] that if $M$ has one end and if $2 \pi \chi(M)-c(M)<2 \pi$, then for every monotone increasing sequence $\left\{K_{j}\right\}$ of compact sets with $\bigcup K_{j}=M$, 


$$
\lim _{j \rightarrow \infty} \frac{\int_{K_{j}} \mathfrak{M} \circ A d M}{\int_{K_{j}} d M}=2 \pi \chi(M)-c(M) .
$$

The proof of this equation essentially depends on the fact that $M$ admits no straight lines. This property is guaranteed by the assumptions on the total curvature and the uniqueness of endpoint of $M$.

It should also be noted that all results mentioned above are obtained under the assumption that $M$ has one endpoint. In the case where $M$ has more than one endpoint (and this is the case where we are interested in this paper), it will be natural to consider that each endpoint shares the value $2 \pi \chi(M)-c(M)$ in the following sense. Let $M$ have $k$ endpoints and let $K \subset M$ be a compact set with the property that $M \backslash \operatorname{Int}(K)$ consists of $k$ tubes $U_{1}, \ldots, U_{k}$ such that each $U_{i}$ is homeomorphic to $S^{1} \times[0, \infty)$ and that each $\partial U_{i}$ is a piecewise smooth simply closed curve. Then the Gauss-Bonnet theorem states that $c(K)+\sum_{i=1}^{k} \kappa\left(\partial U_{i}\right)=2 \pi \chi(M)$, where $c(K)=\int_{K} G d M$ and $\kappa\left(\partial U_{i}\right)$ denotes the curvature integral over the boundary curve $\partial U_{i}$. For each $i=1, \ldots, k$ the value

$$
s_{i}(M):=\kappa\left(\partial U_{i}\right)-c\left(U_{i}\right)
$$

is nonnegative and independent of the choice of tube. Moreover

$$
\sum_{i=1}^{k} s_{i}(M)=2 \pi \chi(M)-c(M) .
$$

For details see [15]. Thus one observes that each endpoint corresponding to $U_{i}$ shares the value $2 \pi \chi(M)-c(M)$.

With these notations our main results will be stated as follows.

Theorem A. Assume that $M$ admits total curvature and has $k$ endpoints. If $s_{i}(M) \leq 2 \pi$ holds for each $i=1, \ldots, k$, then for every monotone increasing sequence $\left\{K_{j}\right\}$ of compact sets with $\bigcup K_{j}=M$,

$$
\begin{aligned}
\operatorname{Min}_{1 \leq i \leq k} s_{i}(M) & \leq \lim _{j \rightarrow \infty} \inf \frac{\int_{K_{j}} \mathfrak{M} \circ A d M}{\int_{K_{J}} d M} \\
& \leq \lim _{j \rightarrow \infty} \sup \frac{\int_{K_{j}} \mathfrak{M} \circ A d M}{\int_{K_{J}} d M} \leq \operatorname{Max}_{1 \leq i \leq k} s_{i}(M) .
\end{aligned}
$$

Theorem B. Assume that $M$ admits total curvature and has $k$ endpoints. Let $\mathfrak{C}$ be a simply closed smooth curve in $M$ and let $B(t):=\{x \in M ; d(x, \mathfrak{C}) \leq t\}$ and $S(t):=\{x \in M ; d(x, \mathfrak{C})=t\}$, 
where $d$ is the distance function induced from Riemannian metric. If $s_{i}(M) \leq 2 \pi$ holds for each $i=1, \ldots, k$, then

$$
\lim _{t \rightarrow \infty} \frac{\int_{B(t)} \mathfrak{M} \circ A d M}{\int_{B(t)} d M}= \begin{cases}\frac{\sum_{i=1}^{k} s_{i}^{2}(M)}{2 \pi \chi(M)-c(M)} & \text { if } 2 \pi \chi(M)-c(M)>0, \\ 0 & \text { if } 2 \pi \chi(M)-c(M)=0 .\end{cases}
$$

REMARK 1. Shiohama first proved an inequality in Theorem B under the stronger assumption that $s_{i}(M)<2 \pi$. But subsequent improvement on the asymptotic behavior of $\mathfrak{M} \circ A$ was obtained by Shioya and Tanaka. It turns out that the existence of straight lines on $M$ is no objection at all. Tanaka's proof for the asymptotic behavior of $\mathfrak{M} \circ A$ by assuming $s_{i}(M)=2 \pi$ will be provided in Lemma 1.1 . Shioya has extended this result to the case where $+\infty \geq s_{i}(M) \geq 2 \pi$. This result will be published independently because the proof is fascinating and of independent interest in itself.

REMARK 2. Theorem B does not hold for any monotone increasing sequence $\left\{K_{j}\right\}$ of compact sets with $\bigcup K_{j}=M$. For example, consider a surface $M$ of revolution in $\mathbb{R}^{3}$ : Let $f: \mathbb{R} \rightarrow(0, \infty)$ be a positive smooth function satisfying $f(t)=1$ for $t \leq-1, f(t)=$ $(t \cdot \tan \theta+1)$ for $t \geq 1$, where $\theta$ is a constant in $(0, \pi / 2) . M$ is defined as

$$
M=\left\{(x, y, z) \in \mathbb{R}^{3} ; y^{2}+z^{2}=f(x)^{2}, x \in \mathbb{R}\right\} .
$$

Then $s_{1}(M)$ and $s_{2}(M)$ are 0 and $2 \pi \sin \theta$ and $2 \pi \chi(M)-c(M)=$ $2 \pi \sin \theta$. For any given $\varepsilon>0$ there exists a positive number $t_{\varepsilon}$ such that if $p \in M$ satisfies $x(p)<-t_{\varepsilon}$, then $\mathfrak{M} \circ A(p)<\varepsilon$, and such that if $x(p)>t_{\varepsilon}$, then $\mathfrak{M} \circ A(p) \in\left(s_{2}(M)-\varepsilon, s_{2}(M)+\varepsilon\right)$. For an arbitrary fixed number $\alpha>0$ choose a monotone increasing sequence $\left\{K_{j}^{\alpha}\right\}$ of compact sets of $M$ with $\bigcup K_{j}^{\alpha}=M$ such that

$$
\operatorname{Area}\left\{p \in K_{j}^{\alpha} ; x(p)>0\right\} / \operatorname{Area}\left\{p \in K_{j}^{\alpha} ; x(p)<0\right\}=\alpha .
$$

Then, computation will show that

$$
\lim _{j \rightarrow \infty} \frac{\int_{K_{j}^{\alpha}} \mathfrak{M} \circ A d M}{\int_{K_{j}^{\alpha}} d M}=\frac{s_{1}(M)+\alpha s_{2}(M)}{\alpha+1}=\frac{(2 \pi \chi(M)-c(M)) \alpha}{\alpha+1} .
$$

Since $\alpha>0$ is arbitrary, this example will suggest the validity of Theorem A. 
1. Preliminaries. Let $K \subset M$ be a compact set with the property that $M \backslash \operatorname{Int}(K)$ consists of $k$ tubes $U_{1}, \ldots, U_{k}$ such that each $\partial U_{i}$ is a piecewise smooth closed curve. For a point $p \in M \backslash \operatorname{Int}(K)$ taken sufficiently away from $K, A(p)$ is divided into two subsets $A_{K}(p)$ and $A_{K}^{\prime}(p)$ as follows: For $u \in A(p)$ set $\gamma_{u}(t):=\exp _{p} t u, t \geq 0$.

$$
\begin{aligned}
& A_{K}(p):=\left\{u \in A(p) ; \gamma_{u}([0, \infty)) \cap K \neq \varnothing\right\}, \\
& A_{K}^{\prime}(p):=\left\{u \in A(p) ; \gamma_{u}([0, \infty)) \cap \operatorname{Int}(K)=\varnothing\right\} .
\end{aligned}
$$

Both $A_{K}(p)$ and $A_{K}^{\prime}(p)$ are closed in $S_{p}(1)$. It follows from minimizing property of rays emanating from $p$ that $A_{K}(p) \cap A_{K}^{\prime}(p)$ consists of at most two elements. Therefore

$$
\mathfrak{M} \circ A(p)=\mathfrak{M} \circ A_{K}(p)+\mathfrak{M} \circ A_{K}^{\prime}(p) .
$$

It was proved in $\S \S 2$ and 3 in [14] that if $0 \leq s_{i}(M)<2 \pi$, then for any given $\varepsilon>0$ there exists an $R(\varepsilon)$ such that for every $p \in U_{i}$ with $d(p, K)>R(\varepsilon)$

$$
s_{i}(M)-\varepsilon \leq \mathfrak{M} \circ A_{K}^{\prime}(p) \leq s_{i}(M)+\varepsilon .
$$

A crucial step of the proof of Theorems A and B is to obtain the asymptotic behavior of $\mathfrak{M} \circ A$. What is left for this purpose is to prove for all $i=1, \ldots, k$ and for all $p \in U_{i}$ with $d(p, K)>R(\varepsilon)$, $(* *)$ $\mathfrak{M} \circ A_{K}(p)<\varepsilon$

and the following

LEMma 1.1 (Tanaka). Assume that $s_{i}(M)=2 \pi$. Then there exists a compact set $K$ with the property that for any $\varepsilon>0$ there exists an $R_{i}(\varepsilon)>0$ such that if $p \in U_{i}$ satisfies $d(p, K)>R_{i}(\varepsilon)$, then

$$
\mathfrak{M} \circ A_{K}^{\prime}(p)>2 \pi-\varepsilon .
$$

Making use of a slightly extended version of an idea developed in the proof of Theorem $\mathrm{C}$ in [12], (**) is proved for a more general closed subinterval $S_{p}(D(p))$ of $S_{p}(1)$ which contains $A_{K}(p)$. For $p \in U_{i}$ and for $u, v \in A_{K}(p)$ let $D_{u, v}(p)$ be the disk domain in $U_{i}$ bounded by the subarcs of $\gamma_{u}$ and $\gamma_{v}$ between $p=\gamma_{u}(0)=\gamma_{v}(0)$ and their first intersections with $K$ and a subarc of $\partial U_{i}$ between them. Let $D(p)$ be the maximal disk domain among $\left\{D_{u, v}(p): u, v \in A_{K}(p)\right\}$ and $S_{p}(D(p)) \subset S_{p}(1)$ the set of all unit vectors at $p$ tangent to $D(p)$. Define an angle

$$
\theta_{K}(p):=\mathfrak{M}\left(S_{p}(D(p))\right)
$$

Then the proof of $(* *)$ is a direct consequence of the following. 
LEMMA 1.2 (Shioya). Let $K \subset M$ be as above and assume that $s_{i}(M) \leq+\infty$ holds for all $i=1, \ldots, k$. For any $\varepsilon>0$ there exists an $R(\varepsilon)>0$ such that if $p \in M \backslash K$ satisfies $d(p, K)>R(\varepsilon)$, then

$$
\theta_{K}(p)<\varepsilon
$$

2. Proof of Theorems $A$ and $B$ by assuming Lemmas 1.1 and 1.2. First of all consider the case where the total area of $M$ is bounded. Then a slight modification of Lemma 3.1 in [14] implies that there exist $k$ distinct Busemann functions on $M$, each of which corresponds to an endpoint of $M$. A Busemann function is differentiable except a set of measure zero since it is Lipschitz continuous. This fact means that there exists a measure zero set $E$ on $M$ such that $A(p)$ for every $p \in M \backslash E$ consists of exactly $k$ elements. Furthermore one has $2 \pi \chi(M)-c(M)=0$ if the total area of $M$ is bounded (see Theorem 12 in [5] and Corollary of Theorem $A$ in [13]). Therefore the proof of theorems in this case is complete.

Assume that the total area of $M$ is unbounded. Let

$$
R(\varepsilon):=\operatorname{Max}_{1 \leq i \leq k} R_{i}(\varepsilon) .
$$

Let $a$ be the area of closed $R(\varepsilon)$-ball around $K$ and $b$ the integral of $\mathfrak{M} \circ A$ over this closed ball. It follows from $(*)$, Lemmas 1.1 and 1.2 that for all sufficiently large $j$

$$
\begin{aligned}
& \frac{b+\left(\operatorname{Min}_{1 \leq i \leq k} s_{i}(M)-\varepsilon\right)\left\{\int_{K_{J}} d M-a\right\}}{\int_{K_{J}} d M} \\
& \quad \leq \frac{\int_{K_{j}} \mathfrak{M} \circ A d M}{\int_{K_{j}} d M} \leq \frac{b+\left(\operatorname{Max}_{1 \leq i \leq k} s_{i}(M)+\varepsilon\right)\left\{\int_{K_{j}} d M-a\right\}}{\int_{K_{j}} d M} .
\end{aligned}
$$

The proof of Theorem A is complete since $\varepsilon$ is any and the total area of $M$ is unbounded.

For the proof of Theorem B one applies the Fiala-Hartman type isoperimetric inequality which was refined by Shiohama in [12] and [13]. Fix a compact set $K$ containing $\mathfrak{C}$ as in Lemmas 1.1 and 1.2. For every $i=1, \ldots, k$ and for sufficiently large $t>0$ let $L_{i}(t)$ and $A_{i}(t)$ be the length of $S(t) \cap U_{i}$ and the area of $B(t) \cap U_{i}$. Because $M$ admits total curvature $S(t) \cap U_{i}$ is homeomorphic to a circle for all large $t$ (see Theorem B in [13]), and is piecewise smooth for almost all $t$. Note that $A_{i}(t)-A_{i}\left(t^{\prime}\right)=\int_{t^{\prime}}^{t} L_{i}(u) d u$. For every $i=1, \ldots, k$

$$
\lim _{t \rightarrow \infty} \frac{L_{i}(t)}{t}=\lim _{t \rightarrow \infty} \frac{2 A_{i}(t)}{t^{2}}=s_{i}(M) \text {. }
$$


By choosing $R(\varepsilon)$ sufficiently large so as to fulfil

$$
s_{i}(M)-\varepsilon<\frac{L_{i}(t)}{t}<s_{i}(M)+\varepsilon
$$

for all $i=1, \ldots, k$ and for all $t>R(\varepsilon)$, one obtains

$$
\begin{gathered}
\frac{b+\sum_{i=1}^{k}\left(s_{i}(M)-2 \varepsilon\right)\left(s_{i}(M)-\varepsilon\right)^{\left(t^{2}-R(\varepsilon)^{2}\right) / 2}}{\sum_{i=1}^{k}\left(s_{i}(M)+\varepsilon\right)^{\left(t^{2}-R(\varepsilon)^{2}\right) / 2}+a} \leq \frac{\int_{B(t)} \mathfrak{M} \circ A d M}{\int_{B(t)} d M} \\
\leq \frac{b+\sum_{i=1}^{k}\left(s_{i}(M)+2 \varepsilon\right)\left(s_{i}(M)+\varepsilon\right)^{\left(t^{2}-R(\varepsilon)^{2}\right) / 2}}{\sum_{i=1}^{k}\left(s_{i}(M)-\varepsilon\right)^{\left(t^{2}-R(\varepsilon)^{2}\right) / 2}+a} .
\end{gathered}
$$

This completes the proof of Theorem B.

3. Proof of Lemmas. A general formula for the mass of rays emanating from a point $p \in M$ is obtained by using an idea developed by Shiga in [10]. This is stated as

$(* * *)$

$$
\mathfrak{M} \circ A(p)=2 \pi \chi(M)-c\left(M \backslash F_{p}\right),
$$

where $F_{p}:=\left\{\exp _{p} t u ; u \in A(p), t \geq 0\right\}$. This formula plays an essential role for the proof of Lemma 1.1.

For the proof of $(* * *)$ fix a point $p \in M$ and let $T>0$ be a sufficiently large number such that $S(p, T):=\{x \in M ; d(p, x)=$ $T\}$ consists of $k$ piecewise smooth closed curves $C_{1}, \ldots, C_{k}$ in $U_{1}, \ldots, U_{k}$ and such that the break points $x_{i, 1}, \ldots, x_{i, m(i)}$ of $C_{i}$ are joined to $p$ by exactly two distinct minimizing geodesics $\alpha_{i, 1}^{-}$, $\alpha_{i, 1}^{+}, \ldots, \alpha_{i, m(i)}^{-}, \alpha_{i, m(i)}^{+}$with $\alpha_{i, m}^{-}(0)=\alpha_{i, m}^{+}(0)=p, \alpha_{i, m}^{-}(T)=$ $\alpha_{i, m}^{+}(T)=x_{i, m}$ and $x_{i, m}$ is not conjugate to $p$ along $\alpha_{i, m}^{-}$and $\alpha_{i, m}^{+}$. This is possible whenever $T$ is taken to be a sufficiently large non-exceptional value (see [4], [13]). Let $F_{i, m}(i=1, \ldots, k$, $1 \leq m \leq m(i))$ be a disk domain surrounded by $\alpha_{i, m}^{+}([0, T])$, the smooth subarc of $S(p, T)$ with terminal points $x_{i, m}$ and $x_{i, m+1}$ and $\alpha_{i, m+1}^{-}([0, T])$, and $\theta_{i, m}$ the angle between $-\dot{\alpha}_{i, m}^{-}(T)$ and $-\dot{\alpha}_{i, m}^{+}(T)$. If $\kappa_{i, m}$ is the curvature integral of the subarc on $\partial F_{i, m} \cap S(p, T)$, then

$$
c\left(F_{i, m}\right)=\mathfrak{M}\left(S_{p}\left(F_{i, m}\right)\right)-\kappa_{i, m} .
$$

If $B(p, T)$ is the closed $T$-ball around $p$, then

$$
c(B(p, T))+\sum_{i=1}^{k} \sum_{m=1}^{m(i)} \kappa_{i, m}-\sum_{i=1}^{k} \sum_{m=1}^{m(i)} \theta_{i, m}=2 \pi \chi(M) .
$$


It follows from construction that $\bigcup_{i} \bigcup_{m} S_{p}\left(F_{i, m}\right)$ is monotone decreasing with $T$ and converges to $A(p)$ as $T \rightarrow \infty$. The proof of $(* * *)$ is complete since $\lim _{T \rightarrow \infty} \sum_{i=1}^{k} \sum_{m=1}^{m(i)} \theta_{i, m}=0$ (see Theorem C, [12]) and $\lim _{T \rightarrow \infty} c\left(B(p, T) \backslash \bigcup_{i} \bigcup_{m} F_{i, m}\right)=c\left(M \backslash F_{p}\right)$.

Proof of Lemma 1.1. For a compact set $C$ such that $M \backslash C$ consists of $k$ tubes, we choose a $K$ containing $C$ such that every minimizing geodesic joining points in $C$ does not meet $\partial K$. Let $M_{i}$ be a complete open 2-manifold having one end with the properties that there exists an isometric embedding $l$ of $K \cup U_{i}$ into $M_{i}$ and that $M_{i} \backslash l\left(K \cup U_{i}\right)$ consists of $k-1$ disks. From construction it follows that $2 \pi \chi\left(M_{i}\right)-c\left(M_{i}\right)=s_{i}(M)$ and $\chi\left(M_{i}\right)=\chi(M)+(k-1)$. Without loss of generality one may identify points in $U_{i}$ with those images in $M_{i}$ as well as other objects. For $p \in U_{i}$ let $A_{i}(p), A_{K, i}(p)$ and $A_{K, i}^{\prime}(p)$ be the set of all unit vectors tangent to rays on $M_{i}$ from $p$ with the same properties as defined in $M$. Then $A_{K, i}^{\prime}(p)=A_{K}^{\prime}(p)$ follows from the choice of $K$. There is no strict relationship between $A_{K, i}(p)$ and $A_{K}(p)$. But both of them will be estimated in Lemma 1.2. Since $\mathfrak{M} \circ A(p)=\left(\mathfrak{M} \circ A_{K}(p)-\mathfrak{M} \circ A_{K, i}(p)\right)+\mathfrak{M} \circ A_{i}(p)$ and the first term in the right-hand side turns out to be small by Lemma 1.2 , one only needs to show that $\mathfrak{M} \circ A_{i}(p)>2 \pi-\varepsilon$ if $p$ is taken sufficiently away from $K$ in $M_{i}$.

From now on one identifies $M_{i}$ with $M$. For any $\varepsilon>0$ let $K_{\varepsilon} \subset M$ be a compact set containing $K$ such that

$$
\int_{M \backslash K_{\varepsilon}}|G| d M<\varepsilon
$$

By means of $(* * *)$ it suffices for the proof of Lemma 1.1 to show $c\left(M \backslash F_{p}\right)<c(M)+5 \varepsilon$ for $p \in M$ with $d(p, K)>R(\varepsilon)$. It follows from finite connectivity of $M$ that there are at most finitely many non-overlapping sectors $V_{1}(p), \ldots, V_{l}(p)$ in $M$ with the following properties: (1) $V_{j}(p) \cap K_{\varepsilon} \neq \varnothing,(2) \partial V_{j}(p)$ consists of two rays emanating from $p,(3) V_{j}(p)$ is homeomorphic to a closed half-plane, and (4) every ray emanating from $p$ is contained in some $V_{j}(p)$ if it intersects $K_{\varepsilon} . V_{j}(p)$ has the property that if $V_{j}^{\prime}(p) \subset V_{j}(p)$ is a subsector such that there is no ray emanating from $p$ and passing through a point on $\operatorname{Int}\left(V_{j}^{\prime}(p)\right)$, then $c\left(V_{j}^{\prime}(p)\right)=\mathfrak{M}\left(S_{p}\left(V_{j}^{\prime}(p)\right)\right)$. Let $\left\{p_{n}\right\}$ be a divergent sequence of points in $M \backslash K_{\varepsilon}$ such that $\left\{V_{j}\left(p_{n}\right)\right\}$ for each $j=1, \ldots, l$ has a limit $V_{j}$ as $n \rightarrow \infty$. This $V_{j}$ is a strip if it has a nonempty interior. If $V_{j}^{\prime} \subset V_{j}$ is a substrip such that there exists no straight line contained entirely in $\operatorname{Int}\left(V_{j}^{\prime}\right)$, then $c\left(V_{j}^{\prime}\right)=0$. 
Set $V=V_{1} \cup \cdots \cup V_{l} . \quad c\left(M \backslash F_{p_{n}}\right) \leq c\left(K_{\varepsilon}\right)-c\left(K_{\varepsilon} \cap F_{p_{n}}\right)+\varepsilon$ and $\left\{c\left(K_{\varepsilon} \cap F_{p_{n}}\right)\right\}_{n}$ tends to $c\left(K_{\varepsilon} \cap V\right)$ as $n \rightarrow \infty$. Thus for all sufficiently large numbers $n, c\left(M \backslash F_{p_{n}}\right) \leq c(M \backslash V)+4 \varepsilon$. Since $V_{j}$ is a strip, a result of Cohn-Vossen (see Satz 3, [2]) implies that $c\left(V_{j}\right) \leq 0$ for all $j=1, \ldots, l$. This implies that $c\left(M \backslash V_{j}\right) \leq 2 \pi \chi\left(M \backslash V_{J}\right)-4 \pi$. But since $\chi\left(M \backslash V_{j}\right)=\chi(M)+1$ the above inequality reduces to $c\left(M \backslash V_{j}\right) \leq 2 \pi \chi(M)-2 \pi$. It follows from the assumption for $c(M)$ that $c\left(M \backslash V_{J}\right) \leq c(M)$, and in particular $c\left(V_{j}\right)=0$ for all $j=$ $1, \ldots, l$. Therefore $c\left(M \backslash F_{p_{n}}\right) \leq c(M \backslash V)+4 \varepsilon \leq c(M)+5 \varepsilon$. This together with $(* * *)$ proves Lemma 1.1.

Proof of Lemma 1.2. A contradiction will be derived by supposing that there exists a divergent sequence $\left\{p_{n}\right\}$ of points such that $\theta_{K}\left(p_{n}\right) \geq \varepsilon_{0}$ holds for all $n$ and for some $\varepsilon_{0}>0$. Without loss of generality we may consider that $\left\{p_{n}\right\}$ is contained in a tube $U$.

To derive a contradiction consider the universal Riemannian covering $\widetilde{U}$ of $U$ whose covering projection is denoted by $\pi$. Let $\tau:[0, \infty) \rightarrow M$ be a ray emanating from a point on $\partial U$ such that $\tau([0, \infty))$ is contained entirely in $U$. Cut open $U$ along $\tau([0, \infty))$ and let $\widetilde{U}_{-1}, \widetilde{U}_{0}, \widetilde{U}_{1}, \ldots$ be the fundamental domains of $U$ lying in this order in $\widetilde{U}$. Let $\tilde{\tau}_{i}:[0, \infty) \rightarrow \widetilde{U}$ be the lifted ray of $\tau$ such that its image lies in $\partial \widetilde{U}_{i-1} \cap \partial \widetilde{U}_{i}$ and $\widetilde{W}:=\widetilde{U}_{0} \cup \widetilde{U}_{1} \cup \widetilde{U}_{2}$. Then $\partial \widetilde{W}$ consists of two rays $\tilde{\tau}_{0}([0, \infty)), \tilde{\tau}_{3}([0, \infty))$ and a subarc of $\partial \widetilde{U}$ whose terminal points are $\tilde{\tau}_{0}(0)$ and $\tilde{\tau}_{3}(0)$.

The intersection of the two minimizing segments on $\partial D\left(p_{n}\right)$ with $\partial U$ will be denoted by $x_{n}$ and $y_{n}$. Set $D_{n}=D\left(p_{n}\right)$ and let $\tilde{p}_{n}:=$ $\pi^{-1}\left(p_{n}\right) \cap \widetilde{U}_{1}$ and $\widetilde{D_{n}} \subset \widetilde{U}$ the lift up of $D_{n}$ satisfying $\tilde{p}_{n} \in \partial \widetilde{D}_{n}$. Let $\tilde{x}_{n}:=\pi^{-1}\left(x_{n}\right) \cap \partial \widetilde{D}_{n}$ and $\tilde{y}_{n}:=\pi^{-1}\left(y_{n}\right) \cap \partial \widetilde{D}_{n}$. It follows from minimizing property of rays that the lifted minimizing geodesics joining $\tilde{p}_{n}$ to $\tilde{x}_{n}$ and $\tilde{p}_{n}$ to $\tilde{y}_{n}$ intersect $\pi^{-1}(\tau)$ at most at one point. This fact means that these geodesics are in $\widetilde{W}$, and in particular, $\tilde{x}_{n}$ and $\tilde{y}_{n}$ are on $\partial \widetilde{W} \cap \partial \widetilde{U}$. By choosing a subsequence, if necessary, one may consider that $\left\{\tilde{x}_{n}\right\},\left\{\tilde{y}_{n}\right\}$ and $\left\{\widetilde{D}_{n}\right\}$ converge to $\tilde{x}, \tilde{y}$ and to an unbounded domain $\widetilde{D}$ in $\widetilde{W}$. Two cases occur in the convergence of $\left\{\widetilde{D}_{n}\right\}$. In the first case, assume that $\left\{\tilde{p}_{n}\right\}$ is contained in the closure of $\widetilde{D}$. Then one may consider that $\left\{\widetilde{D}_{n}\right\}$ is monotone increasing and $\cup \widetilde{D}_{n}=\widetilde{D}$. A slight modification of Theorem $\mathrm{C}$ in [12] implies that $\left\{\theta_{K}\left(p_{n}\right)\right\}$ converges to 0 , a contradiction. In the second case, assume that $\left\{\tilde{p}_{n}\right\}$ is not contained in the closure of $\widetilde{D}$. Without loss of generality one may consider that the lifted minimizing geodesic joining 
$\tilde{p}_{n}$ to $\tilde{x}_{n}$ intersects $\partial \widetilde{D}$ at a point $\tilde{r}_{n}$. Set $\widetilde{E}_{n}:=\widetilde{D}_{n} \backslash \widetilde{D}$ and let $\alpha_{n} \in(0, \pi)$ be the angle at $\tilde{r}_{n}$ of the corner of $\widetilde{D}_{n} \cap \widetilde{D}$. By construction, $\left\{\tilde{r}_{n}\right\}$ contains a divergent subsequence. Then Cohn-Vossen's argument (see $\S 5,[2])$ implies that $\left\{\alpha_{n}\right\}$ has a limit 0 . Let $K_{\varepsilon} \subset M$ be a compact set so as to satisfy

$$
\int_{M \backslash K_{\varepsilon}} G_{+} d M<\varepsilon
$$

Then the area of $\pi^{-1}\left(K_{\varepsilon} \cap U\right) \cap \widetilde{E_{n}}$ tends to zero as $n \rightarrow \infty$ and the curvature integral over $\widetilde{E}_{n} \backslash \pi^{-1}\left(K_{\varepsilon} \cap U\right)$ is bounded above by $\varepsilon$. These facts together with the Gauss-Bonnet theorem for $\widetilde{E_{n}}$ imply that $\left\{\theta_{K}\left(p_{n}\right)\right\}$ contains a subsequence converging to 0 as $n \rightarrow \infty$, a contradiction. This completes the proof of Lemma 1.2.

\section{REFERENCES}

[1] S. Cohn-Vossen, Kürzeste Wege und Totalkrümmung auf Flächen, Compositio Math., 2 (1935), 63-133.

[2] __ Totalkrümmung und geodätische Linien auf einfach zusammenhängenden offenen volständigen Flächenstücken, Recueil Math. Moscow, 43 (1936), 139163.

[3] F. Fiala, La problème isoperimètres sur les surface ouvretes à courbure positive, Comment. Math. Helv., 13 (1941), 293-346.

[4] P. Hartman, Geodesic parallel coordinates in the large, Amer. J. Math., 86 (1964), 705-727.

[5] A. Huber, On subharmonic functions and differential geometry in the large, Comment. Math. Helv., 32 (1957), 13-72.

[6] M. Maeda, On the existence of rays, Sci. Rep. Yokohama Nat. Univ., 26 (1979), $1-4$.

[7] _ A geometric significance of total curvature on complete open surfaces, Geometry of Geodesics and Related Topics, Advanced Studies in Pure Math., 3 (1984), 451-458, Kinokuniya, Tokyo, 1984.

[8] __ On the total curvature of noncompact Riemannian manifolds II, Yokohama Math. J., 33 (1985) 93-101.

[9] T. Oguchi, Total curvature and measure of rays, Proc. Fac. Sci. Tokai Univ., 21 (1986), 1-4.

[10] K. Shiga, On a relation between the total curvature and the measure of rays, Tsukuba J. Math., 6 (1982), 41-50.

[11] _ A relation between the total curvature and the measure of rays II, Tohoku Math. J., 36 (1984), 149-157.

[12] K. Shiohama, Cut locus and parallel circles of a closed curve on a Riemannian plane admitting total curvature, Comment. Math. Helv., 60 (1985), 125-138.

[13] _ Total curvatures and minimal areas of complete open surfaces, Proc. Amer. Math. Soc., 94 (1985), 310-316.

[14] __ An integral formula for the measure of rays on complete open surfaces, $\mathbf{J}$. Differential Geometry, 23 (1986), 197-205. 
[15] K. Shiohama and M. Tanaka, An isoperimetric problem for infinitely connected complete open surfaces, preprint.

[16] T. Shioya, On asymptotic behavior of the mass of rays, Proc. Amer. Math. Soc., 108 (1990), 495-505.

Received February 4, 1988.

KYUSHU UNIVERSITY

FUKUOKA, 812-JAPAN

AND

TOKAI UNIVERSITY

HiRATSUKA, 259-12-JAPAN 


\section{PACIFIC JOURNAL OF MATHEMATICS EDITORS}

\author{
V. S. VARADARAJAN \\ (Managing Editor) \\ University of California \\ Los Angeles, CA 90024-1555-05 \\ Herbert Clemens \\ University of Utah \\ Salt Lake City, UT 84112 \\ ThOMAs ENRIGHT \\ University of California, San Diego \\ La Jolla, CA 92093
}

R. FINN

Stanford University

Stanford, CA 94305

HeRmanN FlaschKa

University of Arizona

Tucson, AZ 85721

VAUGHAN F. R. JoNES

University of California

Berkeley, CA 94720

SteVen KerCKhofF

Stanford University

Stanford, CA 94305
ROBION KIRBY

University of California

Berkeley, CA 94720

C. C. Moore

University of California

Berkeley, CA 94720

HAROLD STARK

University of California, San Diego

La Jolla, CA 92093

\section{ASSOCIATE EDITORS}
R. ARENS
E. F. BECKENBACH
B. H. NeumanN
F. Wolf
K. YoshidA
(1906-1982)
(1904-1989)

\section{SUPPORTING INSTITUTIONS}

UNIVERSITY OF ARIZONA
UNIVERSITY OF BRITISH COLUMBIA
CALIFORNIA INSTITUTE OF TECHNOLOGY
UNIVERSITY OF CALIFORNIA
MONTANA STATE UNIVERSITY
UNIVERSITY OF NEVADA, RENO
NEW MEXICO STATE UNIVERSITY
OREGON STATE UNIVERSITY
UNIVERSITY OF ARIZONA
UNIVERSITY OF BRITISH COLUMBIA
UNIVERSITY OF CALIFORNIA
MONTANA STATE UNIVERSITY
NEW MEXICO STATE UNIVERSITY

\author{
UNIVERSITY OF OREGON \\ UNIVERSITY OF SOUTHERN CALIFORNIA \\ STANFORD UNIVERSITY \\ UNIVERSITY OF HAWAII \\ UNIVERSITY OF TOKYO \\ UNIVERSITY OF UTAH \\ WASHINGTON STATE UNIVERSITY \\ UNIVERSITY OF WASHINGTON
}

The Supporting Institutions listed above contribute to the cost of publication of this Journal, but they are not owners or publishers and have no responsibility for its content or policies.

Mathematical papers intended for publication in the Pacific Journal of Mathematics should be in typed form or offset-reproduced (not dittoed), double spaced with large margins. Please do not use built up fractions in the text of the manuscript. However, you may use them in the displayed equations. Underline Greek letters in red, German in green, and script in blue. The first paragraph must be capable of being used separately as a synopsis of the entire paper. In particular it should contain no bibliographic references. Please propose a heading for the odd numbered pages of less than 35 characters. Manuscripts, in triplicate, may be sent to any one of the editors. Please classify according to the 1980 Mathematics Subject Classification (1985 Revision) scheme which can be found in the December index volumes of Mathematical Reviews. Supply name and address of author to whom proofs should be sent. All other communications should be addressed to the managing editor, or Elaine Barth, University of California, Los Angeles, California 90024-1555-05.

There are page-charges associated with articles appearing in the Pacific Journal of Mathematics. These charges are expected to be paid by the author's University, Government Agency or Company. If the author or authors do not have access to such Institutional support these charges are waived. Single authors will receive 50 free reprints; joint authors will receive a total of 100 free reprints. Additional copies may be obtained at cost in multiples of 50 .

The Pacific Journal of Mathematics is issued monthly as of January 1966. Regular subscription rate: $\$ 190.00$ a year (6 Vols., 12 issues). Special rate: $\$ 95.00$ a year to individual members of supporting institutions.

Subscriptions, orders for numbers issued in the last three calendar years, and changes of address should be sent to Pacific Journal of Mathematics, P.O. Box 969, Carmel Valley, CA 93924, U.S.A. Old back numbers obtainable from Kraus Periodicals Co., Route 100, Millwood, NY 10546.

The Pacific Journal of Mathematics at P.O. Box 969, Carmel Valley, CA 93924 (ISSN 0030-8730) is published monthly. Second-class postage paid at Carmel Valley, California 93924, and additional mailing offices. Postmaster: send address changes to Pacific Journal of Mathematics, P.O. Box 969, Carmel Valley, CA 93924.

\section{PUBLISHED BY PACIFIC JOURNAL OF MATHEMATICS, A NON-PROFIT CORPORATION}




\section{Pacific Journal of Mathematics}

\section{Vol. 143, No. $2 \quad$ April, 1990}

Gustavo Corach, Horacio Porta and Lázaro Recht, Differential geometry of systems of projections in Banach algebras ................. 209

Peter Fleischmann and Jens Carsten Jantzen, Simple periodic modules of

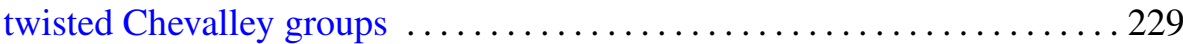

Niels Gronbaek, Amenability of discrete convolution algebras, the commutative case ...................................243

Nguyên H. V. Hung, The mod 2 equivariant cohomology algebras of

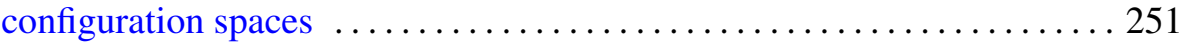

Wojciech Kucharz, Global almost analytic algebraicity of analytic sets . . . 287 John Merrill, A class of consistent anti-Martin's axioms .............. 301

Takafumi Murai, The power 3/2 appearing in the estimate of analytic

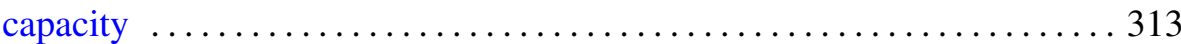

L. Panaitopol and Doru Stefanescu, On the generalized difference

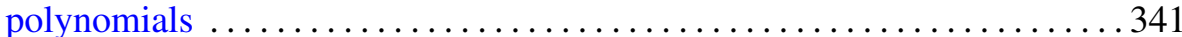

Katsuhiro Shiohama, Takashi Shioya and Minoru Tanaka, Mass of rays

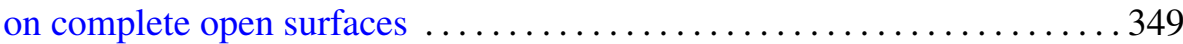

Gerhard Ströhmer, About compressible viscous fluid flow in a bounded

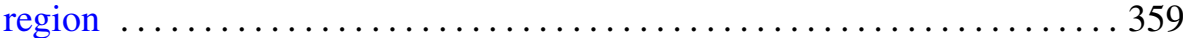

A. Ülger, Arens regularity sometimes implies the RNP 